\title{
Crystal structure of $1,1^{\prime}$-dibenzylferrocene, $\mathrm{C}_{24} \mathrm{H}_{22} \mathrm{Fe}$
}

\author{
J. J. Byrne, A. Rebello, P. Y. Chavant, M.-Th. Averbuch-Pouchot and Y. Vallée \\ Université Joseph Fourier, L.E.D.S.S., U.M.R. C.N.R.S. 5616. BP 53, 38041 Grenoble Cedex 9. France
}

Received April 11. 1997, CSD-No. 409002

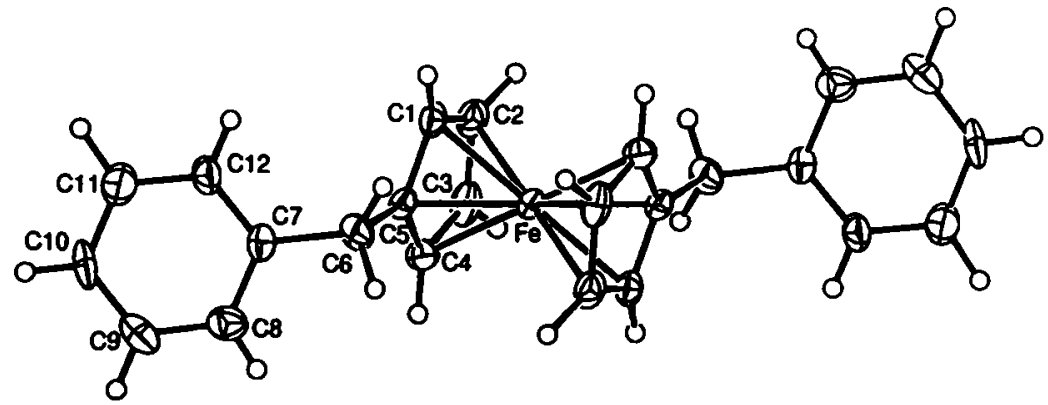

$\mathrm{C}_{24} \mathrm{H} 22 \mathrm{Fe}$, monoclinic, $P 12 \mathrm{~V} / a \mathrm{l}$ (No. 14), $a=15.804(9) \AA, b=6.034(4) \AA, c=9.437(6) \AA$, $\beta=97.79(1)^{\circ}, V=891.6 \AA^{3}, Z=2, R(F)=0.054$, $R_{\mathrm{w}}(F)=0.064$.

Source of material: The reduction of $1,1^{\prime}$-dibenzoylferrocene by dimethylsulfide-borane in THF with the CBS catalyst was previously described (see ref. 1) to yield the corresponding diol in high eniantiomeric excess. On replacement of THF by toluene, the overreduction of the ketone functions has resulted in the title compound.

The Fe atom of the molecular unit, located on an inversion center, is surrounded by a centrosymmetric neighborhood of ten $\mathrm{C}$ atoms at distances ranging from $2.025(7) \AA$ to $2.048(7) \AA$.

Table 1. Parameters used for the X-ray data collection

\begin{tabular}{ll}
\hline Crystal: & yellow plate, size $0.10 \times 0.35 \times 0.56 \mathrm{~mm}$ \\
Wavelength: & Mo $K_{\alpha}$ radiation $(0.7107 \AA)$ \\
$\mu:$ & $8.71 \mathrm{~cm}^{-1}$ \\
Diffractometer: & Enraf-Nonius CAD4 \\
Scan mode: & $\omega$ \\
$T_{\text {measuremen: }}$ & $293 \mathrm{~K}$ \\
$2 \theta_{\text {max: }}$ & $40^{\circ}$ \\
$\mathrm{N}\left(h k l_{\text {unique: }}\right.$ & 652 \\
Criterion for $I_{0}:$ & $l_{0}>3 \sigma\left(I_{0}\right)$ \\
N(param)refined: & 160 \\
Programs: & SAPI 91, teXsan \\
\hline
\end{tabular}

Table 2. Final atomic coordinates and displacement parameters (in $\AA^{2}$ )

\begin{tabular}{|c|c|c|c|c|c|}
\hline Atom & Site & $x$ & $y$ & $z$ & $U_{\text {iso }}$ \\
\hline$H(1)$ & $4 e$ & $0.129(4)$ & $0.78(1)$ & $0.044(7)$ & $0.04(2)$ \\
\hline$H(2)$ & $4 e$ & $-0.028(6)$ & $0.90(2)$ & $0.11(1)$ & $0.12(4)$ \\
\hline$H(3)$ & $4 e$ & $-0.075(5)$ & $0.56(1)$ & $0.245(7)$ & $0.06(2)$ \\
\hline$H(4)$ & $4 e$ & $0.034(5)$ & $0.26(2)$ & $0.239(8)$ & $0.08(3)$ \\
\hline$H\left(6^{\prime}\right)$ & $4 e$ & $0.176(4)$ & $0.20(1)$ & $0.075(6)$ & $0.04(2)$ \\
\hline$H\left(6^{\prime \prime}\right)$ & $4 e$ & $0.196(4)$ & $0.44(1)$ & $0.022(6)$ & $0.03(2)$ \\
\hline$H(8)$ & $4 e$ & $0.228(4)$ & $0.02(1)$ & $0.290(6)$ & $0.03(2)$ \\
\hline$H(9)$ & $4 e$ & $0.317(5)$ & $-0.02(2)$ & $0.495(8)$ & $0.07(3)$ \\
\hline$H(10)$ & $4 e$ & $0.421(4)$ & $0.25(1)$ & $0.542(6)$ & $0.04(2)$ \\
\hline$H(11)$ & $4 e$ & $0.418(6)$ & $0.60(2)$ & $0.411(9)$ & $0.11(3)$ \\
\hline$H(12)$ & $4 e$ & $0.314(4)$ & $0.61(1)$ & $0.213(7)$ & $0.05(2)$ \\
\hline
\end{tabular}

\section{References}

1. Schiwk, L.; Knochel P.: A new practical asymmetric synthesis of $\mathrm{C}_{2}$-symmetrical 1,1'-ferrocenyl diols via CBS-reduction. Tetrahedron Lett. 37 (1996) 25-28.

2. Fan Hai-Fu: Structure Analysis Programs with Intelligent Control (SAPI91). Rigaku Corporation, Tokyo, Japan 1991.

3. Molecular Structure Corporation: teXsan. Single crystal structure analysis software. Version 1.7. MSC, 3200 Research Forest Drive, The Woodlands, TX 77381, USA 1995.

4. Zachariasen, W. H.: A general theory of X-ray diffraction in crystal. Acta Crystallogr. 23 (1967) 558-564.

Table 3. Final atomic coordinates and displacement parameters (in $\AA^{2}$ )

\begin{tabular}{|c|c|c|c|c|c|c|c|c|c|c|}
\hline Atom & Site & $x$ & $y$ & $z$ & $U_{11}$ & $U_{22}$ & $U_{33}$ & $U_{12}$ & $U_{13}$ & $U_{23}$ \\
\hline $\mathrm{Fe}$ & $2 c$ & 0 & $1 / 2$ & 0 & $0.0269(8)$ & $0.0324(9)$ & $0.0315(8)$ & $-0.0030(8)$ & $0.0015(6)$ & $-0.0058(8)$ \\
\hline$C(1)$ & $4 e$ & $0.0952(5)$ & $0.700(1)$ & $0.0909(7)$ & $0.035(4)$ & $0.047(5)$ & $0.030(4)$ & $-0.006(4)$ & $-0.003(3)$ & $-0.001(4)$ \\
\hline$C(2)$ & $4 e$ & $0.0170(5)$ & $0.761(1)$ & $0.1383(8)$ & $0.047(5)$ & $0.046(5)$ & $0.045(4)$ & $0.000(5)$ & $-0.006(4)$ & $-0.015(4)$ \\
\hline$C(3)$ & $4 e$ & $-0.0123(5)$ & $0.574(1)$ & $0.2078(7)$ & $0.042(5)$ & $0.072(7)$ & $0.030(4)$ & $0.002(5)$ & $0.001(4)$ & $-0.021(4)$ \\
\hline$C(4)$ & $4 e$ & $0.0484(5)$ & $0.402(1)$ & $0.2025(8)$ & $0.045(5)$ & $0.036(5)$ & $0.039(4)$ & $-0.005(4)$ & $0.005(4)$ & $0.001(4)$ \\
\hline$C(5)$ & $4 e$ & $0.1139(4)$ & $0.478(1)$ & $0.1304(7)$ & $0.039(4)$ & $0.035(5)$ & $0.032(4)$ & $-0.008(4)$ & $-0.004(3)$ & $-0.005(4)$ \\
\hline$C(7)$ & $4 e$ & $0.2577(4)$ & $0.323(1)$ & $0.2297(7)$ & $0.024(4)$ & $0.052(6)$ & $0.029(4)$ & $0.001(4)$ & $0.006(3)$ & $-0.002(4)$ \\
\hline $\mathrm{C}(8)$ & $4 e$ & $0.2582(5)$ & $0.136(2)$ & $0.3160(9)$ & $0.051(5)$ & $0.040(6)$ & $0.061(5)$ & $0.004(5)$ & $0.012(4)$ & $0.000(5)$ \\
\hline$C(9)$ & $4 e$ & $0.3172(6)$ & $0.111(2)$ & $0.4351(9)$ & $0.058(6)$ & $0.060(6)$ & $0.053(5)$ & $0.016(5)$ & $0.009(4)$ & $0.024(5)$ \\
\hline$C(10)$ & $4 e$ & $0.3759(5)$ & $0.275(2)$ & $0.4718(8)$ & $0.030(4)$ & $0.093(7)$ & $0.041(5)$ & $0.013(5)$ & $-0.014(4)$ & $0.012(5)$ \\
\hline$C(11)$ & $4 e$ & $0.3747(5)$ & $0.463(2)$ & $0.3893(9)$ & $0.050(5)$ & $0.070(7)$ & $0.053(5)$ & $-0.009(5)$ & $-0.003(4)$ & $0.003(5)$ \\
\hline$C(12)$ & $4 e$ & $0.3157(5)$ & $0.482(2)$ & $0.2705(8)$ & $0.030(4)$ & $0.057(6)$ & $0.050(5)$ & $0.002(5)$ & $-0.007(4)$ & $0.009(5)$ \\
\hline
\end{tabular}

